\title{
Planar Evanescent Wave Ammonia Sensor - Influence of Refractive Index Profile on Sensor Properties
}

\author{
P. Karasiński*, C. Tyszkiewicz And R. Rogoziński \\ Department of Optoelectronics, Silesian University of Technology \\ B. Krzywoustego 2, 44-100 Gliwice, Poland
}

\begin{abstract}
The paper presents the results of the investigations involving the influence of the change of launching conditions on the characteristics of the planar amplitude evanescent wave ammonia sensors. Investigated sensors are based on the channel gradient waveguides, which are produced with the application of the ion exchange in glasses. In our investigations we have tested two sensor structures of different refractive index profiles. The absorption sensitive films were produced using sol-gel technology.
\end{abstract}

PACS: $81.20 . \mathrm{Fw}, 42.70 . \mathrm{Ce}, 07.07 . \mathrm{Df}$

\section{Introduction}

Over the recent years we have been witnessing a very intensive development of planar waveguide chemical sensors. The development in question is stimulated by various factors. On the one hand, it is the increasing demand for new measurement techniques, which help to monitor the condition of our natural environment, to control technological processes or to detect the presence of hazardous substances. On the other hand, we can enjoy the benefits resulting from the application of optical waveguide techniques [1-3]. Planar evanescent wave chemical and biochemical sensors can be realized with application of monomode or multimode waveguides. Monomode waveguides are applied in sensor structures with phase modulation [3-9] and multimode waveguides are applied in sensor structures with amplitude modulation. Monomode planar uniform waveguides can be obtained with the application of plasma-enhanced vapour deposition (PECVD) [10], low-pressure chemical vapour deposition (LPCVD) [11], metal organic chemical vapour deposition (MOCVD) [12], pulsed laser deposition (PLD) [13] or sol-gel technology [3-8, 14-18]. Planar waveguides of great modes quantity can be fabricated by ion exchange in glasses [19-26]. Such waveguides are particularly suitable for applications in technology of evanescent wave chemical sensors with amplitude modulation.

The paper is devoted to planar evanescent wave ammonia sensor with amplitude modulation. The physical effect applied in presented sensor is the absorption change of waveguide cover, caused by ammonia concentration change. The amplitude detection and available light sources, as well as economic calculations speak for the application of multimode waveguides in chemical sensors with absorption sensitive film. The properties of amplitude evanescent wave sensor are determined by the properties of the sensitive film and the properties of the ap-

\footnotetext{
* corresponding author; e-mail: pawel.karasinski@polsl.pl
}

plied waveguide. The sensitive film determines the sensitivity of the sensor, response time and regeneration time, measuring range, influence of temperature and humidity on sensor's response as well as selectivity of sensor's response. A waveguide in multimode systems determines sensor's sensitivity to the changes of launching conditions of the sensor structure, which is the most significant flaw of multimode amplitude systems and is caused by the dependence of attenuation coefficients on the mode's order. This problem can be eliminated or considerably reduced by the application of waveguides having an appropriately selected refractive profile. In such a case we can bring about the same reaction of particular modes with absorption sensitive film. We have reported the realizability of waveguide fabrication of different refractive index profiles in our previous works [21,22]. We widely discussed the influence of the refractive index shape on distribution of modal attenuation in ion-exchange waveguides with absorptive covers in Refs. [21-26].

The paper presents the results of our investigations involving the planar amplitude ammonia sensor. The main goal of this paper is presentation of the influence of the shape of applied waveguide's refractive index profile on the sensitivity of the sensor structure to launching conditions. In the paper we presented experimental results for two kinds of sensor structures. The studies were concentrated on multimode structures whereof waveguides were produced in ion exchange process in glass substrates, and the sensitive films were produced in sol-gel technology. We present the technology as well as the results of our investigations of the fabricated sensors. We also provide transmission characteristics of the sensors and the influence of the change of excitation condition on the obtained characteristics.

\section{Fundamentals and technology}

The diagram of the structure of planar ammonia sensor is presented in Fig. 1. A sensitive film is deposited on a gradient channel waveguide produced in ion exchange 


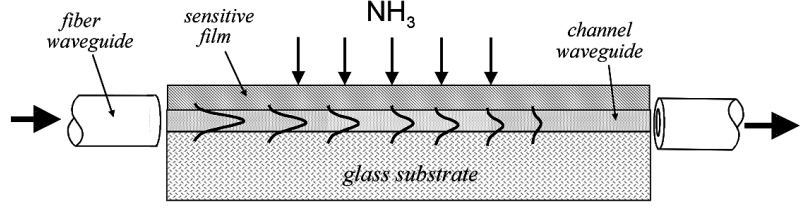

Fig. 1. Structure of the evanescent wave ammonia sensor.

process in glass. The produced planar structures were coupled with standard multimode optic fiber telecommunication waveguides $(62.5 / 125 \mu \mathrm{m})$. Sensitive films in the investigated structures were produced in sol-gel technology $[27,28]$. Silica is a matrix binding the indicator, which was bromocreosole purple. In effect of the reaction of ammonia on the sensitive film, the change of its absorption is taking place, proportional to the concentration of ammonia. Chemical processes taking place in the sensitive film are described by the following reactions:

$$
\begin{aligned}
& \mathrm{NH}_{3} \text { (vapour) }+\mathrm{H}_{2} \mathrm{O} \leftrightarrow \mathrm{NH}_{4}^{+} \mathrm{OH}^{-}, \\
& \mathrm{NH}_{4}^{+} \mathrm{OH}+\mathrm{H}^{+} \mathrm{Dye}^{-} \leftrightarrow \mathrm{NH}_{4}^{+} \mathrm{Dye}^{-}+\mathrm{H}_{2} \mathrm{O}, \\
& \mathrm{NH}_{4}^{+} \mathrm{Dye}^{-} \leftrightarrow \mathrm{H}^{+} \mathrm{Dye}^{-}+\mathrm{NH}_{3} \text { (vapour). }
\end{aligned}
$$

The maximum involving the changes of absorption of the applied sensitive film occurs with the wavelength $\lambda=600 \mathrm{~nm}$ [27]. The modes propagating in the structure and penetrating the sensitive film with the evanescent field are attenuated. If we assume that there is no coupling between the modes within the area of the sensitive film, then the total power propagating in all modes can be presented as follows:

$$
P_{\mathrm{c}}(x)=\sum_{m=0}^{M-1} P_{m}(0) \exp \left(-\tilde{\gamma}_{m} n^{(\mathrm{i})} x\right),
$$

where $m$ is the number of mode, $M$ is the number of all modes, $P_{m}(0)$ is the power incoupled to the $m$-th mode, $\tilde{\gamma}_{m}$ is the normalized attenuation coefficient of the $m$-th mode, $n^{(\mathrm{i})}$ is the imaginary part of the refractive index of sensitive film, $x$ is the length of sensitive film. Sensor structure will be insensitive to the changes of excitation conditions if the values of normalized attenuation coefficients are not dependent on the mode order. As it will be presented below, also non-monotonic distribution of modal attenuation makes possible the reduction of the influence the launching conditions on transmission characteristics of the sensor structure.

Channel waveguides applied in multimode sensor structures were produced using ion exchange $\mathrm{Ag}^{+}-\mathrm{Na}^{+}$ in glass. Substrates made of soda-lime glass and borosilicate glass were applied. In soda-lime glass the structures were produced with the use of dissolved admixture source, which was silver nitrate $\mathrm{AgNO}_{3}$ dissolved in sodium nitrate $\mathrm{NaNO}_{3}$ in molar ratio equal to 0.0025 . In the second case a two-stage process was applied, which involved pre-diffusion in pure silver nitrate and then heat- ing. Selective ion-exchange process in glass substrates was carried out with the use of aluminium masks. Detailed description is given below.

\section{Theoretical analysis}

For the produced sensor structures, theoretical distribution of modal attenuation was determined. Following the approach of the effective index method, the calculations were carried out for planar waveguides having refractive index profiles of channel waveguides in their symmetry plane. In the calculations we assumed complex refractive index of the sensitive film $n_{\mathrm{c}}=n_{\mathrm{c}}^{(\mathrm{r})}-\mathrm{i} n_{\mathrm{c}}^{(\mathrm{i})}$ and we used the expressions on mode attenuation coefficient $\left(\gamma_{m}=\tilde{\gamma}_{m} n_{\mathrm{c}}^{(\mathrm{i})}\right)$ formulated by Snyder and Love [29], Eq. (11-63):

$$
\gamma_{m}=2 k_{0}\left(\frac{\varepsilon_{0}}{\mu_{0}}\right)^{1 / 2} \frac{\int_{-d}^{0} n_{\mathrm{c}}^{(\mathrm{r})} n_{\mathrm{c}}^{(\mathrm{i})}|\boldsymbol{E}|^{2} \mathrm{~d} z}{\operatorname{Re}\left(\int_{-\infty}^{\infty}\left|\left(\boldsymbol{E} \times \boldsymbol{H}^{*}\right)_{x}\right| \mathrm{d} z\right)},
$$

where $\boldsymbol{E}, \boldsymbol{H}$ - vectors, respectively, of electric and magnetic fields, $\varepsilon_{0}, \mu_{0}$ - permittivity and permeability in free space, respectively, $k_{0}=2 \pi / \lambda$ - wave number in free space corresponding to wavelength $\lambda, d-$ sensitive film thickness.

Effective indexes and the distribution of modal fields were determined using the $4 \times 4$ matrix method $[6,30]$.

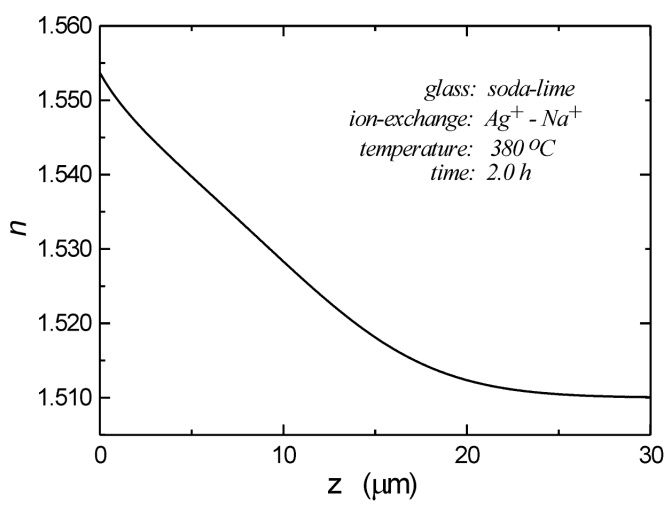

Fig. 2. Refractive index profile of the channel waveguide in their symmetry plane. Ion-exchange from solution $\mathrm{AgNO}_{3}: \mathrm{NaNO}_{3}$ with molar ratio 0.0025. Mask opening width $w=30 \mu \mathrm{m}$.

Figure 2 presents the refractive profile corresponding to the channel waveguide produced in soda-lime glass substrate with the application of dissolved admixture source. The process has been carried out at temperature $380^{\circ} \mathrm{C}$ for $1.5 \mathrm{~h}$.

Figure 3 presents the calculated distributions of modal attenuation for that type of refractive index profile. For both polarizations, the obtained relations are monotonic functions. Together with the rise of mode order $m$, for the described case, the attenuation of modes is decreasing.

Figure 4 presents the refractive profile of the waveguide produced in two-stage technological process in borosilicate glass substrate. The technological process involved 


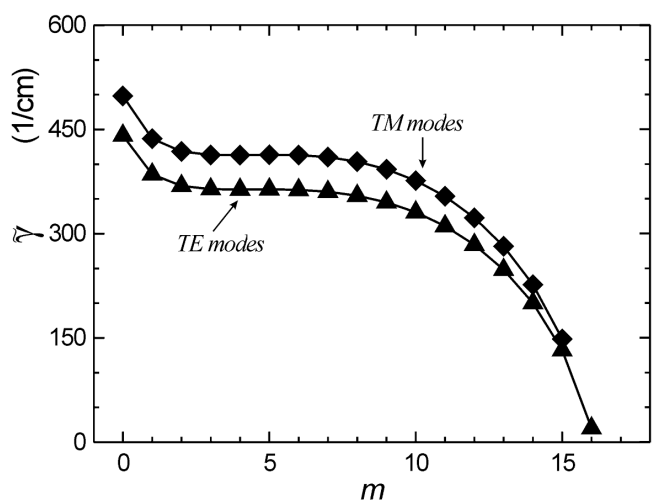

Fig. 3. Dependence of normalized attenuation coefficients on mode order for refractive index profile presented in Fig. 2. Lines are drawn as guide for eye.

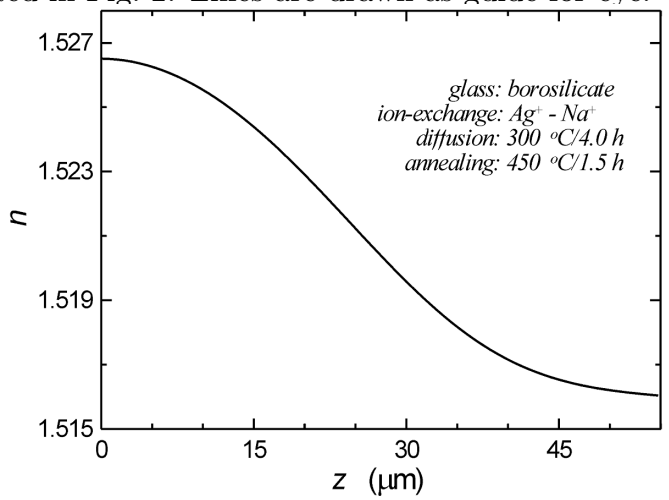

Fig. 4. Refractive index profile of the channel waveguide in their symmetry plane. Ion-exchange from solution $\mathrm{AgNO}_{3}$. Mask opening width $w=20 \mu \mathrm{m}$.

following steps: pre-diffusion at temperature $300^{\circ} \mathrm{C}$ for $4.0 \mathrm{~h}$, and then annealing at temperature $450^{\circ} \mathrm{C}$ for $1.5 \mathrm{~h}$.

The distributions of modal attenuation corresponding to that profile were presented in Fig. 5. In that case, the distributions of modal attenuation have the character of non-monotonic relations. Initially, the normalized coefficients are increasing together with the increase in

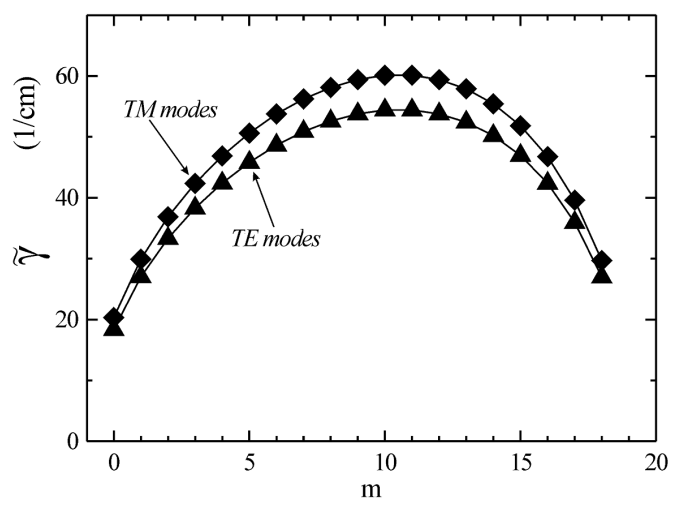

Fig. 5. Dependence of normalized attenuation coefficients on mode order for refractive index profile presented in Fig. 4. Lines are drawn as guide for eye. mode order. For the modes of higher order - higher than the 10th order, normalized attenuation coefficients are decreasing with the increasing in mode order.

\section{Experimental}

\subsection{Measurement setup}

The produced structures of ammonia sensors were investigated in the measurement system whereof diagram is presented in Fig. 6. The investigated sensor structure PS was placed in a measurement chamber K. From the measurement chamber, the waveguide fibers coupled with the structure were guided out. The light source LS (LED; EL 383-2UOC, Elfa) of the wavelength $\lambda=611 \mathrm{~nm}$ was also coupled with the optical fiber, and its connection to the investigated planar structure PS was affected by the optical fiber connector of ST type. In all cases the same light source was applied. The diode LED was fed with alternate signal from the generator G. For the detection of signal we used a homodyne nanovoltmeter and signal recording system RS coupled with a computer. The measurement chamber $\mathrm{K}$ with the sensor structure placed inside was joined with the gas batching system. As ammonia source, we used ammonia solution of the concentration $5000 \mathrm{ppm}$ in synthetic air, and as carrier gas we applied air. Both gases were mixed in a computer controlled batching system MCS, and then they were moistened and passed to the measurement chamber. During the measurements, the light intensity of the diode LED has been controlled. For this purpose we applied the reference circuit, which is not showed in Fig. 6.

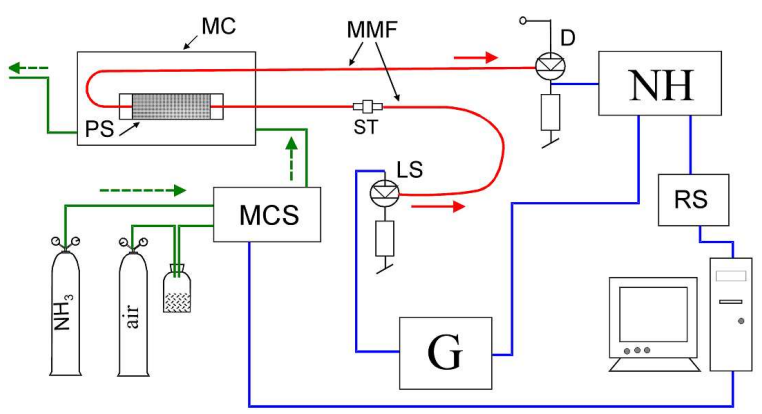

Fig. 6. Diagram of the measurement setup. PS - planar structure, MC - measurement chamber, MMF multimode optical fiber waveguide, ST - optical fiber connector, LS - light source, D - detection diode, MCS - mixing and control batching system, G - generator, $\mathrm{NH}$ - homodyne nanowoltmeter, $\mathrm{RS}$ - recording system.

\subsection{Results}

In the carried out experimental investigations, we determined the influence of the change of excitation conditions of the produced sensor structures on their characteristics. Repeatability of sensor's response was tested 


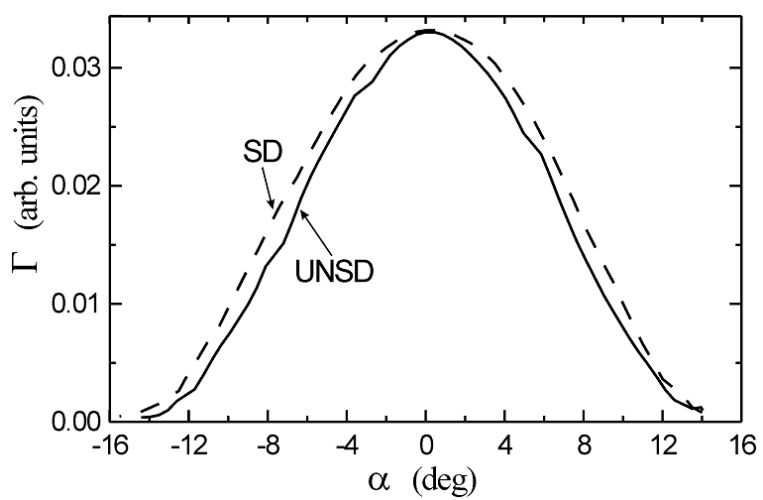

Fig. 7. Distribution of the power density. SD - stable distribution, UNSD - unstable distribution.

and regeneration times of the sensor subjected to the influence of ammonia of different concentration were compared. The obtained results are presented in Fig. 7 .

Transmission characteristics of the produced sensor structures were determined for two different distributions of power in waveguide fiber coupled with the sensor structure. Angular distributions of power density, which were recorded in the far field, are presented in Fig. 7. Unstable distribution of power (UNSD) was recorded when the excitation waveguide was positioned in a rectilinear way between the light source S (Fig. 6) and the planar structure PS. Stable distribution (SD) was recorded when a mode scrambler was applied in the line, which guides the light to the planar structure. It can be seen from the presented relations that in the case of unstable distribution, higher density of power occurs for small angles $\alpha$, which are close to zero. It means that in this case the modes of the lowest order are preferred, as the ones that guide more power than the modes of higher order.

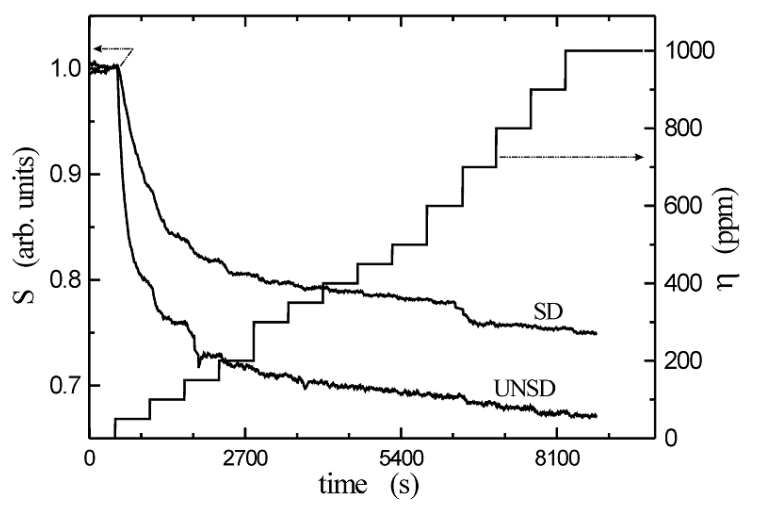

Fig. 8. The characteristics of sensor with waveguides produced in soda-lime glass substrate for different launching conditions.

Transmission characteristics for the investigated sensor structures for different excitation conditions are presented in Figs. 8 and 9.

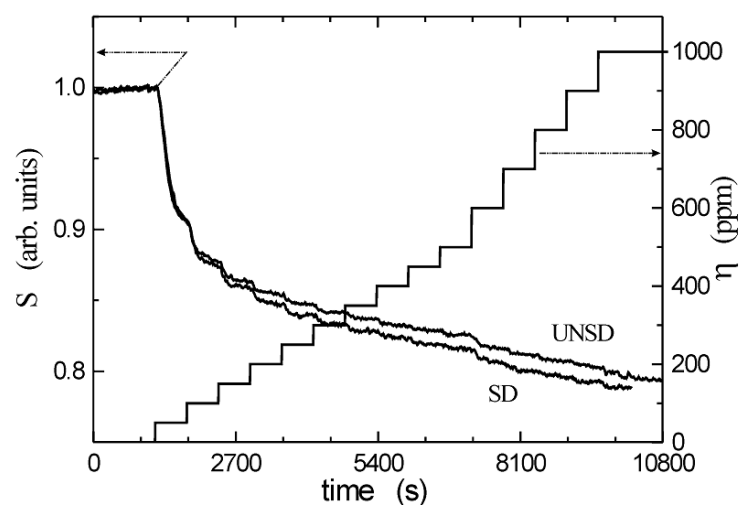

Fig. 9. The characteristics of sensor with waveguides produced in borosilicate glass substrate for different launching conditions.

Figure 8 presents the characteristics of the sensor whose waveguide was produced in soda-lime glass substrate from the dissolved dopant source of molar ratio $\mathrm{AgNO}_{3}: \mathrm{NaNO}_{3}=0.0025$. The characteristics were recorded for gradually increasing concentration $\eta$ of ammonia. The dependence of ammonia concentration $\eta$ in the measurement chamber on time is also presented on this diagram. The characteristic corresponding to the unstable distribution (UNSD) of power shows higher dynamics of changes of signal $S$ than the characteristic corresponding to the stable distribution of power (SD). It results from the monotonic dependence of attenuation coefficients on mode order (Fig. 3). When the sensor structure is excited with the use of UNSD, the modes of lower order are subjected to stronger excitation than in the case of SD. These modes, as it can be seen from Fig. 3, correspond with higher values of attenuation coefficients than for the modes of higher order. Hence, with the change of excitation conditions, when less power is transferred to the lower order modes and more power to the modes of higher order, a relatively high change of sensor's characteristic is observed.

Transmission characteristics of the sensor produced with the application of a waveguide formed in a two-stage technological process in borosilicate glass substrate are presented in Fig. 9. In this case we can observe a small difference in the run of the characteristics, corresponding with different excitation conditions. As it can be seen, this structure shows low sensitivity to the change of excitation conditions. The distribution of modal attenuation for this structure has the character of non-monotonic relation, which, with the applied excitation conditions (Fig. 7), yields a similar run of sensor's characteristics. The reduction of power guided in lower order modes and the increase of power in higher order modes has therefore inconsiderable influence on the run of sensor's characteristics. We can distinguish two ranges on the characteristics presented in Figs. 8 and 9, which have noticeably different dynamics of the changes of signal $S$ with the increase of ammonia concentration $\eta$. Initially, when the 
ammonia concentration does not exceed $\eta=100 \mathrm{ppm}$, very strong changes of signal $S$ are observed with the increase of ammonia concentration. For high ammonia concentration, above $\eta=100 \mathrm{ppm}$, the change in dynamics of signal $S$ is considerably lower.

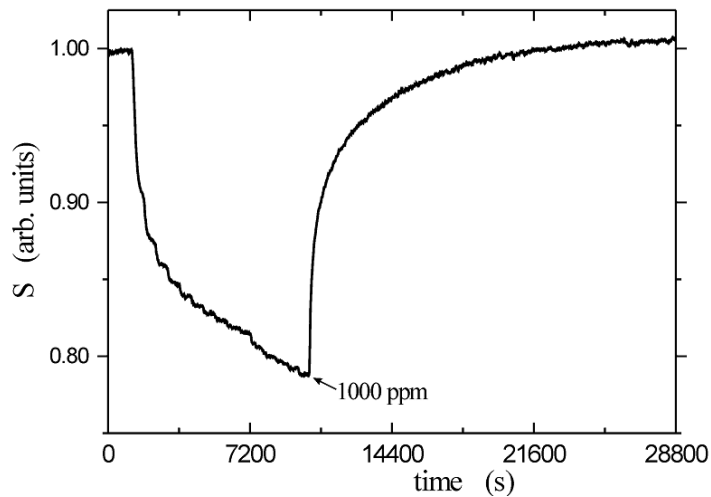

Fig. 10. Response of the sensor to the increasing ammonia concentration and its regeneration.

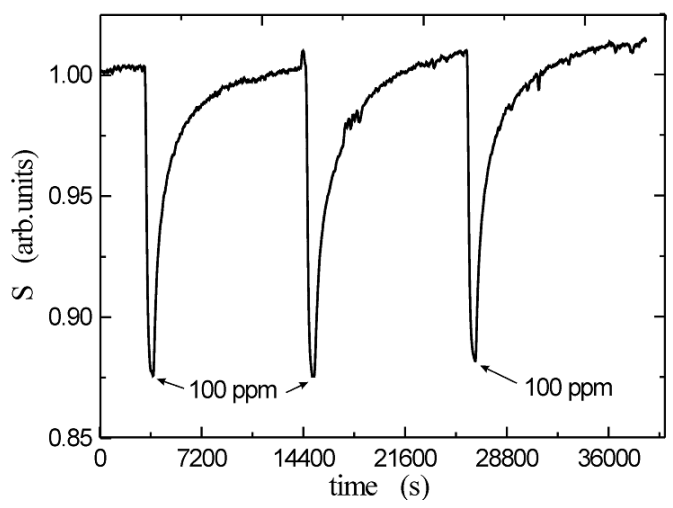

Fig. 11. Repeatability of sensor's responses.

Figure 10 presents the response of the sensor to the gradually increasing ammonia concentration to $1000 \mathrm{ppm}$, and then its regeneration. It can be seen from the presented characteristic that the full regeneration of the sensitive film subjected to the influence of ammonia of the concentration $1000 \mathrm{ppm}$ is taking place after the time of $4 \mathrm{~h}$.

The repeatability of sensor's responses is illustrated by the characteristic presented in Fig. 11. We can see here two change cycles of ammonia concentration in the measurement chamber. In each of them the maximum concentration of ammonia was $\eta=100 \mathrm{ppm}$. The time of full regeneration of sensitive film is in this case shorter than for the concentration $\eta=1000 \mathrm{ppm}$, and equals $3 \mathrm{~h}$. Therefore, we can see that the regeneration time of sensitive film subjected to the influence with ammonia is increasing with increase in ammonia concentration.

The comparison of the responses of the sensor subjected to the influence of ammonia of the concentration

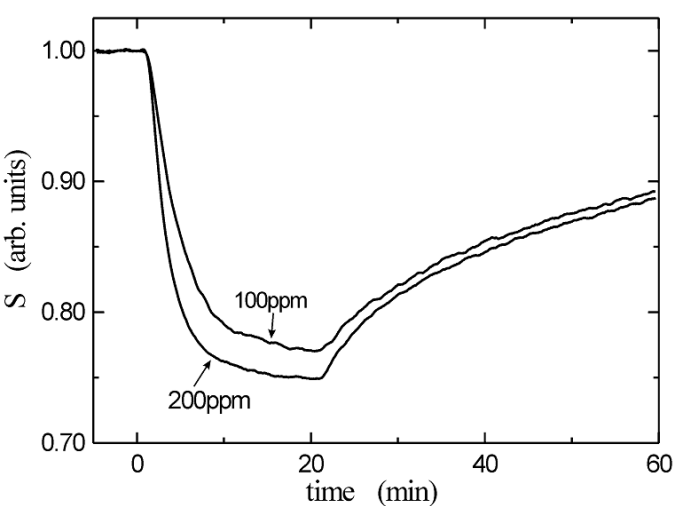

Fig. 12. Response and regeneration of the sensor subjected to the influence of ammonia of different concentration.

$100 \mathrm{ppm}$ and $200 \mathrm{ppm}$ is illustrated in Fig. 12. It can be clearly seen that the signal $S$ is decreasing faster with higher concentration of ammonia. In effect, the time after which the signal reaches the minimum for both concentrations of ammonia is similar. We can also observe that we have longer regeneration time of sensitive film subjected to the influence of ammonia of higher concentration. Without any doubts for a given indicator, the regeneration time of sensitive film depends on its porosity. For sensitive films, which are more porous, we can expect shorter regeneration times.

\section{Conclusions}

The paper presents the results of investigations involving ammonia sensors produced in planar technology. Channel waveguides were produced in ion-exchange process in glass, and sensitive films with the use of sol-gel technique. The setup for the investigation of sensor characteristics was described. We presented the characteristics of sensors produced with the use of different channel waveguides, with different distributions of modal attenuation corresponding to them. For these sensors, the influence of the change of excitation conditions on the obtained characteristics was presented.

The sensor structure with the waveguide produced in soda-lime glass, for which the distribution of modal attenuation was a monotonic relation, was characterized by high sensitivity to the changes of excitation conditions. The structure formed in two-stage technological process, for which the distribution of modal attenuation was a non-monotonic relation, was characterized by low sensitivity to the changes of excitation conditions. The strongest dynamics of changes of the signal was observed for low concentrations of ammonia. By an appropriate selection of refractive index and thickness of the sensitive film as well as the length of interaction area, we can optimize the parameters of the sensor for a required range of ammonia concentration. By the application of sensitive films of high porosity, we can shorten the regeneration 
time. Investigations on the technology of that type are being carried out by us.

\section{Acknowledgments}

The work was partially financed from the research grant of the NCBiR R01 0034 06/2009

\section{References}

[1] B. Culshaw, J. Dakin, Optical Fiber Sensors. Vol. Three - Components and Subsystems, Artech House, Boston-London 1996.

[2] P.V. Lambeck, Measur. Sci. Technol. 17, R93 (2006).

[3] W. Lukosz, Sens. Actuators B 29, 37 (1995).

[4] R. Horváth, G. Fricsovszky, E. Papp, Biosens. Bioelectron. 18, 415 (2003).

[5] P. Karasiński, Proc. SPIE 6348, 63480H (2006).

[6] P. Karasiński, Opto Electron. Rev. 15, 168 (2007).

[7] P. Karasiński, Acta Phys. Pol. A 116, S30 (2009).

[8] P. Karasiński, Opto Electron. Rev. 19, 10 (2011).

[9] A. Ymeti, J. Greve, P.V. Lambeck, T. Wink, S.W.F.M. Hovell, T.A.M. Beumer, R.R. Wijn, R.G. Heideman, V. Subramaniam, J.S. Kanger, Nano Lett. 7, 394 (2002).

[10] Ch. Gorecki, Opt. Lasers Eng. 33, 15 (2000).

[11] P.V. Lambeck, J. van Lith, H.J.W.M. Hoekstra, Sens. Actuators B 113, 718 (2006).

[12] Y. Lou, D.C. Hall, IEEE Sel. Top. Quant. Electron. 11, 1284 (2005).

[13] D.Y. Wang, K.P. Lor, K.K. Chung, H.P. Chan, K.S. Chiang, H.L.W. Chan, C.L. Choy, Thin Solid Films 510, 329 (2006).
[14] C.J. Brinker, G.W. Scherer, Sol-Gel Science, Academic Press, San Diego 1990.

[15] P. Karasiński, Opt. Appl. 34, 467 (2004).

[16] K. Tifenthaler, W. Lukosz, Opt. Lett. 10, 137 (1984).

[17] G.C. Righini, S. Pelli, J. Sol Gel Sci. Technol. 8, 991 (1997).

[18] R.M. Almeida, J. Non-Cryst. Solids 259, 176 (1999).

[19] S.I. Najafi, Introduction to Glass Integrated Optics, Artech House, Boston 1992.

[20] R. Rogoziński, Opt. Commun. 219, 199 (2003).

[21] R. Rogoziński, P. Karasiński, Proc. SPIE 5028, 71 (2003).

[22] R. Rogoziński, P. Karasiński, Opto Electron. Rev. 13, 229 (2005).

[23] P. Karasiński, R. Rogoziński, A. Opilski, Proc. SPIE 4516, 218 (2001).

[24] P. Karasiński, R. Rogoziński, Proc. SPIE 5576, 181 (2004).

[25] P. Karasiński, R. Rogoziński, Opt. Commun. 269, 76 (2007).

[26] P. Karasiński, Opt. Commun. 280, 351 (2007).

[27] P. Karasiński, Opt. Appl. 33, 477 (2003).

[28] C. Tyszkiewicz, P. Karasiński, R. Rogoziński, Acta Phys. Pol. A 118, 1262 (2010).

[29] A.W. Snyder, J.D. Love, Optical Waveguide Theory, Chapman and Hall, London 1983.

[30] P. Karasiński, Proc. SPIE 4239, 229 (2000). 